\title{
Research on the Optimal Combination of Concrete Aggregates Based on Bolomey Equation
}

\author{
Fan Wang ${ }^{1, *}$, Shansuo Zheng ${ }^{2}$, and Bin Wang ${ }^{1}$ \\ ${ }^{1}$ School of Civil \& Architecture Engineering, Xi'an Technological University, Xi'an, 710021, China \\ ${ }^{2}$ School of Civil Engineering, Xi'an University of Architecture and Technology, Xi'an, 710055, China
}

\begin{abstract}
Concrete mixture with high density is one of the important guarantees for its high strength, especially the gradation combination of coarse or fine aggregate. In order to overcome the deficient market supply of sand and gravel for meeting the need of high strength concrete and special concrete, based on the Bolomey equation, combining with the corresponding regulations and grading requirements of the concrete aggregates in the current CHINA standards as follows: Standard for technical requirements and test method of sand and crushed stone (or gravel) for ordinary concrete (JGJ 52), Sand for construction (GB/T 14684), Pebble and crushed stone for construction (GB/T 14685), establish a set of practical numerical calculation methods of the optimal combination on the continuous gradation of concrete aggregates. After concretepreparation test verification, with the optimal combination of Aggregates, concrete mix proportion is easy to determine with higher guarantee rate, its strength can be raised up to $20 \%$ with better durability.
\end{abstract}

\section{Introduction}

Large numbers of studies showed that the aggregate gradation hads important impact on the performance, mechanical properties, long-term performance and durability of concrete. Aggregate gradation could be divided into two categories: continuous-graded gradation and single-graded gradation. Among them, single-graded gradation could also be called discontinuous gradation, which refered to the gradation of taking out one or more intermediate gradations in continuous grading. Some research had shown that gap graded aggregate mixed in the concrete of greater workability can cause concrete segregation easily, which is mixed in the concrete mixture with vibratory compaction and lower workability. Classic theory of the aggregate gradation had much as follows: Maximum density proposed by W. B. Fuller and S. E. Thompson[1], J. Bolomey's grading formula[2], the American C. A. G. Weymouth's particle interference theory[3], thick and surrounding stacking theory proposed by Chinese Liu Chongxi[4], the grain composition fractal theory[5-10]. The above theories were theoretical and inconvenient to apply in practical engineering. Therefore, in order to cope with the sand and stone with uneven quality and different graduation in the market, a set of practical numerical calculation methods of the optimal combination on the continuous gradation of concrete aggregate was proposed based on Bolomey equation.

\section{Bolomey equation}

Bolomey equation $[2,11]$ is one of the continuous grading theories, which was based on the famous particle size distribution computation theory derived from "ideal particles". As for the aggregate particle, the percent passing through the $d$ sieve opening was calculated according to Eq. 1:

$$
P_{p d}=\frac{A-B+(100-A) \times \sqrt{\frac{d}{D_{\max }}}}{100-B} \times 100 \%
$$

Where $P_{\mathrm{pd}} / \%$ is the percent passing through the dsieve opening, and $D_{\max } / \mathrm{mm}$ is Maximum particle size of aggregates, and $B$ is the percentage of colloidal particles of all solid particles, and $A$ is the value of the empirical coefficient depends on the slump of concrete mix and the type of aggregates, as shown in Table 1.

Table 1. Value of A depends on concrete slump and aggregates.

\begin{tabular}{|c|c|c|c|}
\hline \multirow{2}{*}{$\begin{array}{c}\text { Type of aggregate } \\
\text { particles }\end{array}$} & \multicolumn{3}{|c|}{ slump of concrete $(\mathrm{mm})$} \\
\cline { 2 - 4 } & $\leqslant 50$ & $50 \sim 100$ & $\geqslant 150$ \\
\hline natural & 8 & 10 & 12 \\
\hline $\begin{array}{c}\text { artificial or } \\
\text { machine-made }\end{array}$ & 10 & 12 & 14 \\
\hline
\end{tabular}

\footnotetext{
* Corresponding author: ship_wang2955@ sina.com.
} 


\section{Evaluation of particle size distribution}

The particle size distribution or grading of aggregates was evaluated and measured by the sieve analysis test. The results of the sieve analysis could be expressed by the sieve table, the fineness modulus and the sieving graph. Sieve analysis test should be according to Standard for technical requirements and test method of sand and crushed stone (or gravel) for ordinary concrete (JGJ 52), test square hole sieves with the following openings: $80 \mathrm{~mm}, 63 \mathrm{~mm}, 50 \mathrm{~mm}, 40 \mathrm{~mm}, 31.5 \mathrm{~mm}, 25 \mathrm{~mm}$, $20 \mathrm{~mm}, 16 \mathrm{~mm}$ and $10 \mathrm{~mm}, 5 \mathrm{~mm}, 2.5 \mathrm{~mm}$ and $1.25 \mathrm{~mm}$, $630 \mu \mathrm{m}, 315 \mu \mathrm{m}$ and $160 \mu \mathrm{m}$. Among them, nominal diameter less than $5.00 \mathrm{~mm}$ of rock particles is known as sand, nominal diameter greater than $5.00 \mathrm{~mm}$ of rock particles is known as stone. The degree of fineness modulus was calculated according to Eq. 2:

$$
M_{f}=\left(\sum_{i=1}^{n} \beta_{i}\right) / 100
$$

Where $M_{f}$ is the fineness modulus, and $n$ is the number of test square holes sieves used, and $\beta_{i}$ is the cumulative passing sieve residue of stage sieves.

\section{Simplified optimal combination of aggregates}

Concrete works are often poured in the way of pumping, and the slump of concrete is required to be $50 \mathrm{~mm} \sim 150 \mathrm{~mm}$. In addition, gravel particles usually are used to produce concrete. Therefore, the simplified numerical method of the optimal combination of aggregate particles can be applied according to Bolomey equation.

\subsection{Best combination of sand}

At present, the natural sand is widely used in engineering, which is preferable to that $A^{\prime} s$ value is 10 , and Bolomey equation could be simplified as Eq. 3:

$$
P_{p d}=10+\sqrt{1620 d}(\%)
$$

Accordingly, the particle size distribution of the ideal natural sand particles could be calculated. Especially, the cumulative percent passing on sieve size $630 \mu \mathrm{m}$ was $58.1 \%$. According to Standard for technical requirements and test method of sand and crushed stone (or gravel) for ordinary concrete (JGJ 52), the natural sand having ideal particle size distribution belonged to the II -area sand used in concrete preferentially. Furthermore, the fineness modulus of ideal natural sand particles obtained based on the minimum void volume was 2.71 , could be classified into medium sand.

With the importance of environmental protection and the increasing shortage of resources, the application of artificial or machine-made sand had become more and more. As for the artificial or machine-made sand, it was preferable to that $A^{\prime} s$ value is 12 , and Bolomey equation could be simplified as Eq. 4:

$$
P_{p d}=12+\sqrt{1548.8 d}(\%)
$$

Accordingly, the particle size distribution of the ideal artificial or machine-made sand particles could be calculated. Particularly, the cumulative percent passing on sieve size $630 \mu \mathrm{m}$ was $56.8 \%$. According to Standard for technical requirements and test method of sand and crushed stone (or gravel) for ordinary concrete (JGJ 52), the artificial or machine-made sand having ideal particle size distribution also belonged to the II -area sand used in concrete preferentially. Moreover, the fineness modulus of ideal artificial or machine-made sand particles obtained based on the minimum void volume was 2.65 , could be also classified into medium sand.

For two sand of different fineness modulus, the optimum fineness modulus should be close to the ideal sand particle based on the minimum space volume. Through the linear relationship, the combination ratio could be calculated as Eq.5. For three or more types of sand, it also could be obtained by this analogy.

$$
S 1: S 2=\left(\left|M_{f s 2}-M_{f s}\right|\right):\left(\left|M_{f s}-M_{f s 1}\right|\right)
$$

Where $M_{f s}$ is the fineness modulus of the ideal sand, the value for natural sand is 2.71 , the value for artificial or machine-made sand is 2.65 , and $M_{f s i}$ is the fineness modulus of sand, and $S i$ is the mass ratio of sand.

\subsection{Best combination of crushed stone}

Taking the value of $A$ as 12 , the ideal particle size distribution could be calculated. Specially, the cumulative percent passing of continuous grading crushed stone on each sieve size was consistent basically with the relevant quality requirements of Standard for technical requirements and test method of sand and crushed stone (or gravel) for ordinary concrete (JGJ 52).Taking this one step further, the fineness modulus of ideal crushed stone particles obtained based on the minimum void volume was shown in Table 2 .

Table 2. Fineness modulus of ideal crushed stone.

\begin{tabular}{|c|c|c|c|c|c|c|}
\hline$D_{\max } / \mathrm{mm}$ & 10 & 16 & 20 & 25 & 31.5 & 40 \\
\hline$M_{f g}$ & 6.0 & 6.18 & 6.35 & 6.59 & 6.91 & 7.30 \\
\hline
\end{tabular}

For two crushed stone of different fineness modulus, the optimum fineness modulus should be close to the ideal crushed stone particle based on the minimum space volume. Through the linear relationship, the combination ratio could be calculated as Eq.6. For three or more types of crushed stone, it also could be obtained by this analogy.

$$
G 1: G 2=\left(\left|M_{f g 2}-M_{f g}\right|\right):\left(\left|M_{f g}-M_{f g 1}\right|\right)
$$


Where $M_{f g}$ is the fineness modulus of the ideal crushed stone, and $M_{f g i}$ is the fineness modulus of two types of crushed stone respectively, and $G i$ is the mass ratio of sand.

\subsection{Best combination of sand and crushed stone}

Similarly, according to the simplified Bolomey equation, based on the minimum void volume mechanism, the fineness modulus of ideal sand and crushed stone particles was calculated and shown in Table 3, Fig. 1 and Fig. 2.

Table 3. Fineness modulus of ideal sand and crushed stone.

\begin{tabular}{|c|c|c|}
\hline$D_{\max } / \mathrm{mm}$ & natural sand & $\begin{array}{c}\text { artificial or } \\
\text { machine-made sand }\end{array}$ \\
\hline 10 & 3.49 & 3.42 \\
\hline 16 & 4.07 & 3.99 \\
\hline 20 & 4.40 & 4.32 \\
\hline 25 & 4.78 & 4.69 \\
\hline 31.5 & 5.23 & 5.14 \\
\hline 40 & 5.74 & 5.65 \\
\hline
\end{tabular}

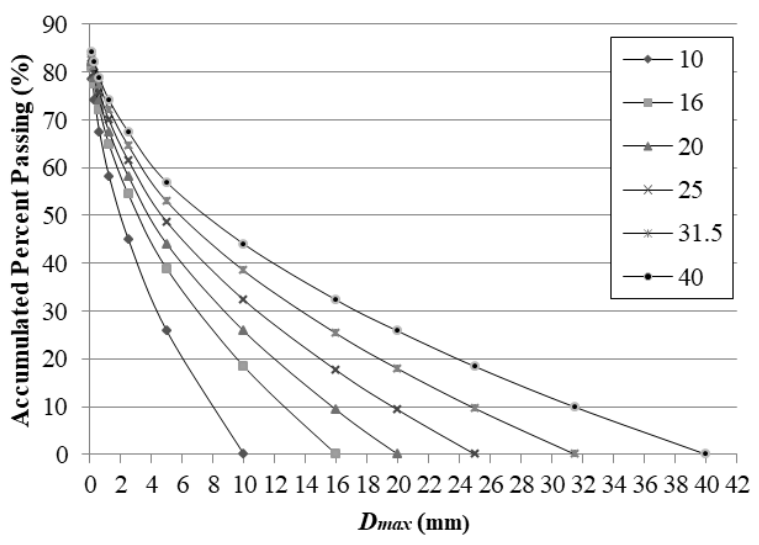

Fig. 1. Sieve analysis results for natural sand and crushed stone.

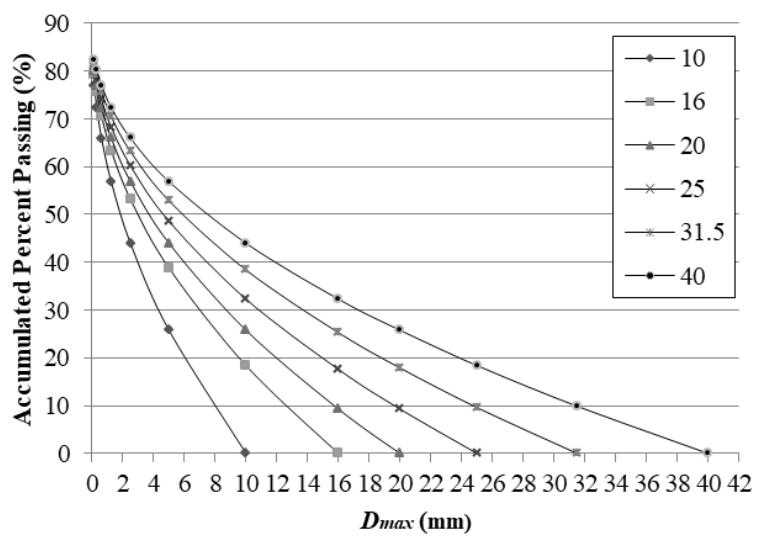

Fig. 2. Sieve analysis results for artificial or machine-made sand and crushed stone

For the optimal combination of sand and crushed stone, whose fineness modulus should be close to the ideal sand and crushed stone. According to the linear relation of the fineness modulus, the combination proportion could be calculated as Eq.7.

$$
S: G=\left(\left|M_{f g}-M_{f}\right|\right):\left(\left|M_{f}-M_{f s}\right|\right)
$$

Where $S$ is the mass ratio of sand, and $G$ is the mass ratio of crushed stone, $M_{f s}$ is the fineness modulus of sand with optimal self-combination first, and $M_{f g}$ is the fineness modulus of crushed stone with optimal selfcombination first.

Moreover, if the slump of concrete was required to be $50 \mathrm{~mm} \sim 150 \mathrm{~mm}$, combining optimally sand with crushed stone for concrete mixture could approach to the combination proportion shown in Table 4.

Table 4. Fineness modulus of ideal crushed stone.

\begin{tabular}{|c|c|c|}
\hline$D_{\max } / \mathrm{mm}$ & natural sand & $\begin{array}{c}\text { artificial or } \\
\text { machine-made sand }\end{array}$ \\
\hline 10 & 3.22 & 2.99 \\
\hline 16 & 1.55 & 1.49 \\
\hline 20 & 1.15 & 1.11 \\
\hline 25 & 0.87 & 0.85 \\
\hline 31.5 & 0.67 & 0.65 \\
\hline 40 & 0.56 & 0.55 \\
\hline
\end{tabular}

\section{Application}

Producing the concrete with strength grade of $\mathrm{C} 70$, the materials used were as follows: Ordinary Portland Cement of 52.5 grading $(\mathrm{P} \bullet \mathrm{O} 52.5 \mathrm{R})$ according with GB/T 21372 and GB 175 on chemical composition, mineral composition and physical properties, natural river sand with fineness modulus of 2.8 and bulk density of $2.6 \mathrm{~g} / \mathrm{cm}^{2}$, continuous grading crushed stone with fineness modulus of 6.6 and maximum particle size of $31.5 \mathrm{~mm}$, non-retarding Polycarboxylates High Performance water-reducing admixture potable water.

The concrete mixtures prepared for use in this study were described in Table 5. The cement content of $460 \mathrm{~kg} / \mathrm{m}^{3}$, w/c of 0.25 and water-reducer's dosage of $2 \%$ remained constant between two mixtures. For the MD-2, $S: G$ is 0.62 through the optimal combination ways in this study.

Table 5. Concrete mixture design.

\begin{tabular}{|c|c|c|c|c|}
\hline $\begin{array}{c}\text { Mixture } \\
\text { designation }\end{array}$ & $\begin{array}{c}\text { Cement } \\
\text { content } \\
\left(\mathrm{kg} / \mathrm{m}^{3}\right)\end{array}$ & $\begin{array}{c}\text { Sand } \\
\left(\mathrm{kg} / \mathrm{m}^{3}\right)\end{array}$ & $\begin{array}{c}\text { Crushed } \\
\text { stone } \\
\left(\mathrm{kg} / \mathrm{m}^{3}\right)\end{array}$ & w/c \\
\hline MD-1 & 460 & 855 & 970 & 0.25 \\
\hline MD-2 & 460 & 700 & 1130 & 0.25 \\
\hline
\end{tabular}

Note: In the naming convention MD is "mixture designation".

Nine groups of separate concrete mixtures were cast for each combination and their compressive strength was measured, shown in Fig. 3. The MD-1 mixture exhibited $78.7 \mathrm{Mpa}$ of maximum compressive strength at day 28 . However, the MD-2 mixture gained $90.6 \mathrm{Mpa}$ of minimum compressive strength at day 28 , increasing strength about $20 \%$ compared to the MD-1 mixture. Obviously, its durability would be better for good compactness. 


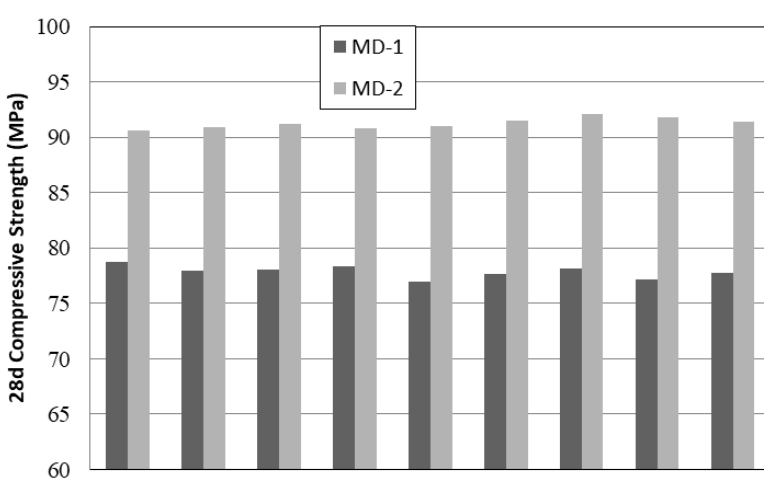

Fig. 3. Compressive strength through 28 days

\section{Conclusions}

Sand or/and crushed stone of any fineness could be combined into ideal graded particles by formula (5-7), which could not only meet the standard requirements, but also overcome the market supply situation that their quality does not good.

Because of well-graded aggregates in the concrete mixture, the fluctuation range of its test mixture ratio was reduced effectively, and its design mixture ratio was more easily determined.

Making full use of the particle gradation effect of concrete aggregates could effectively improve its strength and durability, could also observe high guarantee rate.

\section{Acknowledge}

Assisted by the Project of Shaanxi provincial department of education project (16JK1374).

\section{References}

1. W. B. Fuller, S. E. Thompson, JTD, ASCE, 59, 1:67-143 (1907)

2. Bolomey J., Bulletin, ASCE, 16:22-24(1927)

3. C. A. G. Weymouth, Rock Products, 2:26(1933)

4. Chongxi L.,J.H.E., 2:59-66(1964). (In Chinese)

5. Chongxi L., The Changiang River, 12:34-38(1956). (In Chinese)

6. Feng R., Academic Dissertation, D.L.U.T., 2007. (In Chinese)

7. Qun Y., Zhongyin G., Liping C., J.B.M., 9, 4:419422(2006). (In Chinese)

8. Qianyuan W., Jingshuang H., Rock and Soil Mechanics, 18, 3:93-100(1997). (In Chinese)

9. Qianyuan W., Qing Z., J.G.M.E., 13, 2:109117(1994). (In Chinese)

10. Qianyuan W., Qing Z., J.I.A.E. of Qingdao, 15, 2:61-68(1994). (In Chinese)

11. Fan W., Academic Dissertation, X.A.U.A.T., 2014. (In Chinese) 